\title{
Método não invasivo para predição da pressão arterial com pletismografia e inteligência computacional
}

\author{
Jardel dos Santos Martins* Carlos Anderson Oliveira Silva** \\ Antônio Hamilton Magalhães ${ }^{* * *}$ Felipe Diniz Ramalho ${ }^{* * * *}$ \\ * Instituto Federal do Norte de Minas Gerais, Rodovia BR 367, km 111, \\ Zona Rural, Almenara - MG, (e-mail: jardelmartinsbb@gmail.com) \\ ** Instituto Federal do Norte de Minas Gerais, Rodovia BR 367, km \\ 111, Zona Rural, Almenara-MG, (e-mail: carlos.silva@ifnmg.edu.br) \\ *** Pontifícia Universidade Católica de Minas Gerais, Rua do Rosário, \\ 1081 - Angola,Betim - MG, (e-mail: ahmagalhaes@gmail.com) \\ **** Pontifícia Universidade Católica de Minas Gerais, Av. Itaú, 525 - \\ Dom Cabral, Belo Horizonte - MG, (e-mail: felipedram@gmail.com)
}

\begin{abstract}
Blood Pressure is an important indicator for monitoring and diagnostic of cardiovascular diseases (CD). The World Health Organization (WHO) estimates that CDs accounted for $31 \%$ of deaths by 2015 . Blood Pressure can be measured using various techniques, each having its own methods. Among them, plethysmography (PPG) can be highlighted. This work aims to develop a computational intelligence model capable of estimating systolic and diastolic pressure values, based on the extraction of PPG signal characteristics obtained by a noninvasive method.

Resumo: A pressão arterial é um indicador importante para monitoramento e diagnóstico de doenças cardiovasculares (DC). A Organização Mundial da Saúde (OMS) estima que as DCs foram responsáveis por $31 \%$ das mortes em 2015. A pressão arterial pode ser aferida empregando diversas técnicas, tendo cada uma os seus métodos próprios. Dentre elas, pode-se destacar a pletismografia (PPG). Este trabalho tem por objetivo desenvolver um modelo de inteligência computacional capaz de estimar os valores de pressão sistólica e diastólica, com base na extração de características do sinal PPG obtido por um método não invasivo.
\end{abstract}

Keywords: Plethysmography; Multi Layer Perceptron; Hypertension; Blood Pressure; Palavras-chaves: Pletismografia; Perceptron de Múltiplas Camadas; Hipertensão; Pressão Arterial;

\section{INTRODUÇÃO}

O coração e os vasos sanguíneos constituem o sistema cardiovascular humano e são os principais responsáveis por todo o transporte de gases sanguíneos, nutrientes de alimentos, drogas e etc (Gunasekaran, 2013). As doenças cardiovasculares (DCs) são a causa de um número expressivo de mortes em todo o mundo. A Organização Mundial da Saúde (OMS) estima que as DCs foram responsáveis por $31 \%$ das mortes em 2015, das quais mais de três quartos aconteceram em países de baixa e média renda (WHO, 2018).

As DCs são um grupo de distúrbios dos vasos sanguíneos e coração que incluem (WHO, 2018):

- doença cardíaca coronária - doença dos vasos sanguíneos que suprem o músculo cardíaco;

- doença cerebrovascular - doença dos vasos sanguíneos que alimentam o cérebro;

- doença arterial periférica - doença dos vasos sanguíneos que suprem os braços e pernas;
- doença cardíaca reumática - lesões no músculo cardíaco e nas válvulas cardíacas da febre reumática, causadas por bactérias estreptocócicas;

- cardiopatia congênita - malformações da estrutura cardíaca existentes no nascimento;

- trombose venosa profunda e embolia pulmonar coágulos de sangue nas veias da perna, que podem desalojar e passar para o coração e os pulmões.

Diversos são os motivos que levam às doenças cardiovasculares. Dentre eles, um dos mais significativos é a aterosclerose (placas gordurosas, que se depositam na artéria, bloqueando o natural fluxo sanguíneo), ocasionando uma rigidez arterial, prejudicial ao perfeito funcionamento do aparelho circulatório (WHO, 2018; Corrêa, 2006).

Os fatores de risco comportamentais mais importantes de doença cardíaca e acidente vascular cerebral (AVC) são dieta não saudável, sedentarismo, tabagismo, uso nocivo de álcool e estresse. Os efeitos dos fatores de risco comportamentais podem desencadear fatores de risco intermediários, tais como: hipertensão arterial, aumento da glicose no sangue, aumento de lipídios no sangue, sobrepeso e obesidade. Estes podem ser medidos e indicam se há, 
ou não, algum risco e o seu grau em desenvolver ataques cardíacos, derrames, insuficiências cardíacas dentre outras complicações. Alguns destes pôdem ser mensurados exclusivamente via exames realizados por profissionais da área em hospitais, laboratórios especializados ou unidades de atenção básica à saúde. Porém, a pressão arterial (PA) pode ser aferida empregando diversas técnicas, tendo cada uma os seus métodos e objetivos próprios (Bronzino and Peterson, 2014; Ogedegbe and Pickering, 2010; de Andrade et al., 2015; Lamelas and Jolly, 2015; Banegas et al., 2018). Dentre eles, pode-se destacar a pletismografia (PPG).

Este trabalho tem por objetivo desenvolver um modelo de inteligência computacional capaz de estimar os valores de pressão sistólica e diastólica, com base na extração de características do sinal PPG obtido por um método não invasivo.

O trabalho está organizado em seções. Na seção 2, é apresentada uma revisão bibliográfica sobre o tema. Em seguida, na seção 3, são apresentados os materiais e métodos empregados. Na seção 4 são apresentados e discutidos os resultados alcançados neste trabalho e, por fim, na seção 5 , é feita a conclusão.

\section{REVISÃO BIBLIOGRÁFICA}

Allen, em seu trabalho no ano de 2007, descreve fotopletismografia como uma técnica não invasiva para identificação de mudanças no volume de sangue em nível microvascular, baseada em medições sobre a pele. Adicionalmente, apresenta o formato de um sinal PPG como sendo composto por uma componente variável (AC), pulsativa, que determina variações no volume de sangue, de acordo com o ritmo cardíaco (Allen, 2007). Esta componente é sobreposta a uma componente quase contínua (DC) que varia lentamente influenciada por vários fatores como respiração, atividades do sistema nervoso parassimpático e com processos de regulação térmica. A componente DC representa o volume médio das artérias e a componente AC a parte variável do volume.

Kamshilin e Margaryants descrevem métodos de detecção do sinal PPG, baseado em transmitância e em reflectância. Também mostram o uso de luz na faixa NIR (Near Infrared) para detecção do sinal PPG (Kamshilin and Margaryants, 2017). Song et al. (2009) utilizaram um método de obtenção do sinal PPG com sensor instalado no pulso do paciente, comparando os resultados com os obtidos por meio de esfigmomanômetro. O sinal PPG foi obtido utilizando um arranjo contendo quatro LEDs emissores de luz infravermelha, um em cada canto de um arranjo quadrado, e um fototransistor ao centro. A leitura e digitalização do sinal foram feitas com um microcontrolador MSP 430 da Texas Instruments. A interação da luz com tecido biológico é complexa e envolve múltiplos espalhamentos, absorção, reflexão, transmissão e fluorescência (Allen, 2007).

Do ponto de vista do tratamento dos dados e medição da pressão sistólica e diastólica por meio de PPG, o trabalho desenvolvido por Xing e Sun, utiliza transformada rápida de Fourrier (FFT) para modelagem dos dados (Xing and Sun, 2016). O trabalho elabora um modelo matemático que associa o sinal PPG ao volume da artéria na região compreendida pelo sensor. A comparação é feita entre os sinais PPG e BP (Blood Pressure) com base em componentes espectrais. Um processo de normalização é feito para extração de características. O trabalho descreve o uso de uma rede neural convencional treinada com um conjunto de frequências e fases obtidas por meio da FFT.

Zhang e Feng apresentam um método para estimativa da pressão sistólica e diastólica com base na medição de parâmetros correspondentes ao formato do pulso do sinal PPG, e aplicam este conjunto de parâmetros ao treinamento de uma rede neural (Zhang and Feng, 2017). Também utilizam o método SVM (Support Vector Machine) para modelar e análisar os dados e estimar a pressão sistólica e diastólica de um conjunto de pacientes, efetuando a validação dos dados por medição direta da pressão nestes pacientes.

Kurylyak, Lamonaca e Grimald utilizaram um procedimento semelhante ao utilizado no presente trabalho, baseado na medição das larguras do pulso PPG a 10, 25, 50, 66 e $75 \%$ da amplitude de pico. Estes parâmetros são utilizados para estimar a pressão sistólica e diastólica por regressão linear e também para treinar uma rede neural de múltiplas camadas Kurylyak et al. (2013).

Wang et al. (2017) utilizaram uma câmera RGB para identificar as mudanças na tonalidade da pele como uma forma de monitorar, remotamente, atividades cardíacas, denominado remote PPG. Desenvolveram um modelo matemático relativo a propriedades refletivas da pele e o uso combinado dos canais R, G e B da imagem para obtenção do sinal PPG.

\section{DESENVOLVIMENTO}

Para o desenvolvimento deste trabalho foi utilizada uma base de dados disponibilizada pela Yongbo Liang et al. (2017). O processo de obtenção destes dados é descrito no artigo dos mesmos autores (Liang et al., 2018). A base contém sinais PPG na forma de três arquivos tendo, cada um, 2.1 segundos de amostras do sinal. A taxa de amostragem utilizada foi de $1 \mathrm{kHz}$. A base contém dados de 219 pacientes, registrando também informações como idade, peso, altura, sexo. Aqui descrevemos a forma como os sinais PPG foram obtidos. Foram descartados os casos onde a qualidade do sinal PPG não se mostrou adequada. Os sinais PPG foram obtidos por meio de fotopletismografia e com o uso do sensor instalado no dedo do paciente.

Abaixo são apresentadas as técnicas empregadas no desenvolvimento deste trabalho, abrangendo o pré-processamento dos dados, filtragem, normalização e extração de características.

\subsection{Metodologia}

Para essa base dados, o sinal de PPG foi coletado na dimensão do tempo. Durante a coleta do sinal PPG, foi utilizada uma ponta de prova com sensor SEP9AF2 (SMPLUS Company, Korea). Este sensor utiliza LED duplo nos comprimentos de onda de 660 $\mathrm{nm}$ (Vermelho) e $905 \mathrm{~nm}$ (Infravermelho), taxa de amostragem de $1 \mathrm{kHz}$ e um conversor Analógico-Digital com resolução de 12 - bit. O sinal, a priori, foi condicionado por meio de um filtro 


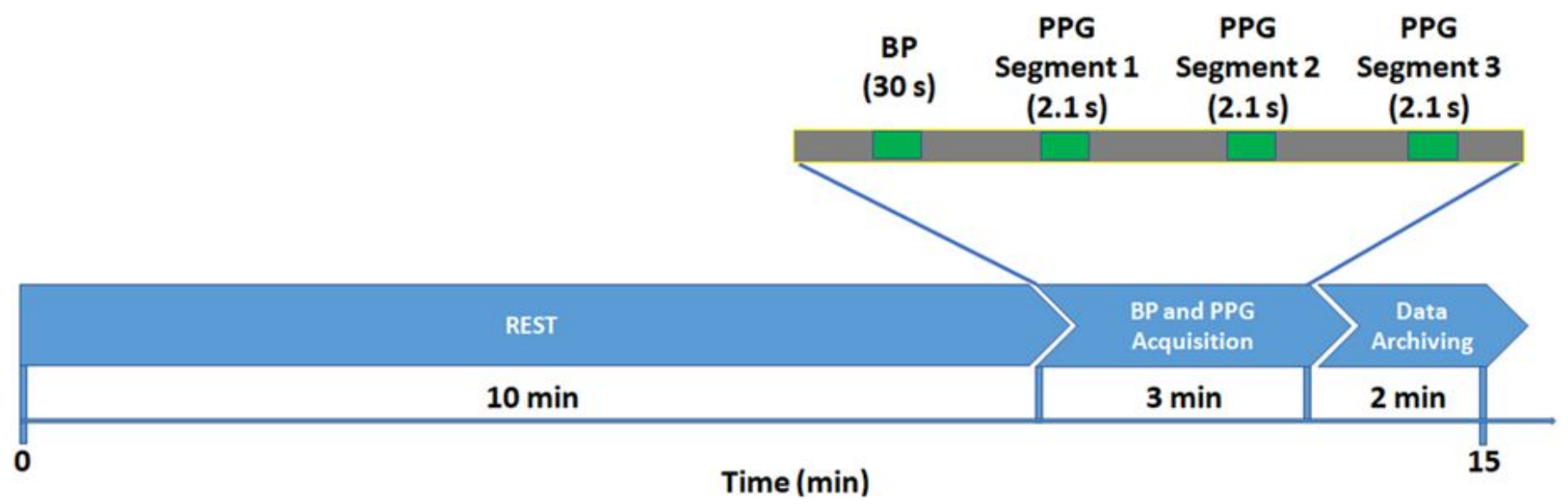

Fig. 1. Diagrama do protocolo de coleta dos dados empregado (Liang et al., 2018).

com banda passante de 0.5 a $12 \mathrm{~Hz}$. Um microcontrolador MSP430FG4618 (Texas Instruments) foi utilizado para coletar os dados amostrados pelo conversor e transmitir, via conexão Bluetooth, para um aplicativo desenvolvido sob a plataforma Android. O protocolo de coleta utilizado para obtenção destes dados foi dividido em dois estágios. No primeiro estágio, o paciente aguardou em repouso por 10 minutos, para estabilização de sua condição fisiológica. Em seguida, iniciou-se o segundo estágio com a aferição da pressão arterial, seguido da coletada dos dados de PPG. Tal atividade foi segmentada em três intervalos de 2.1 segundos, gerando três conjuntos de amostras da forma de onda. O diagrama da Figura 1 apresentada a esquematização do protocolo supracitado.

Pré-processamento O pré-processamento dos dados é uma etapa importante no processo de aprendizado de máquina e interfere diretamente nos resultados obtidos. Esta fase tem por objetivo filtrar ruídos advindos do sensor, do hardware de processamento e eventuais movimentações do paciente. Outro aspecto é normalizar o sinal, uma vez que o mesmo contém uma parte pulsante sobreposta a um componente quase contínuo. Ademais, outra fonte de possíveis ruídos no processamento dos dados é devido à ocorrência de outliers, sendo necessário um processamento que garanta uma equanimidade dos dados. $\mathrm{O}$ processo inicia-se com o escalonamento para o intervalo $[0,1]$. Num segundo momento, a fim de diminuir dados ruidosos que por ventura possam persistir, os valores são filtrados conforme a Equação (1):

$$
x_{t}=\alpha * x_{t-1}+(1-\alpha) * x_{t},
$$

onde $\alpha$ é um fator de escalonamento do algoritmo de filtragem recursiva e $t$ o $t$-ésimo instante de tempo no intervalo do segmento. $\mathrm{O}$ valor de $\alpha$ utilizado foi de 0.95 .

Extração de características As características a serem utilizadas no treinamento dos algoritmos são obtidos da forma de onda do sinal PPG. O sinal PPG, por ser uma forma de onda pulsante (AC), sobreposta a um componente quase contínuo (DC), caracteriza-se por possuir uma série de valores de máximos e mínimos em cada segmento coletado. Para extração das características das ondas, os segmentos de cada pulso foi dividido horizontalmente em quartis (da Cunha and Carvajal, 2009). Os valores de máximos estão acima do primeiro quartil. Os valores mín-

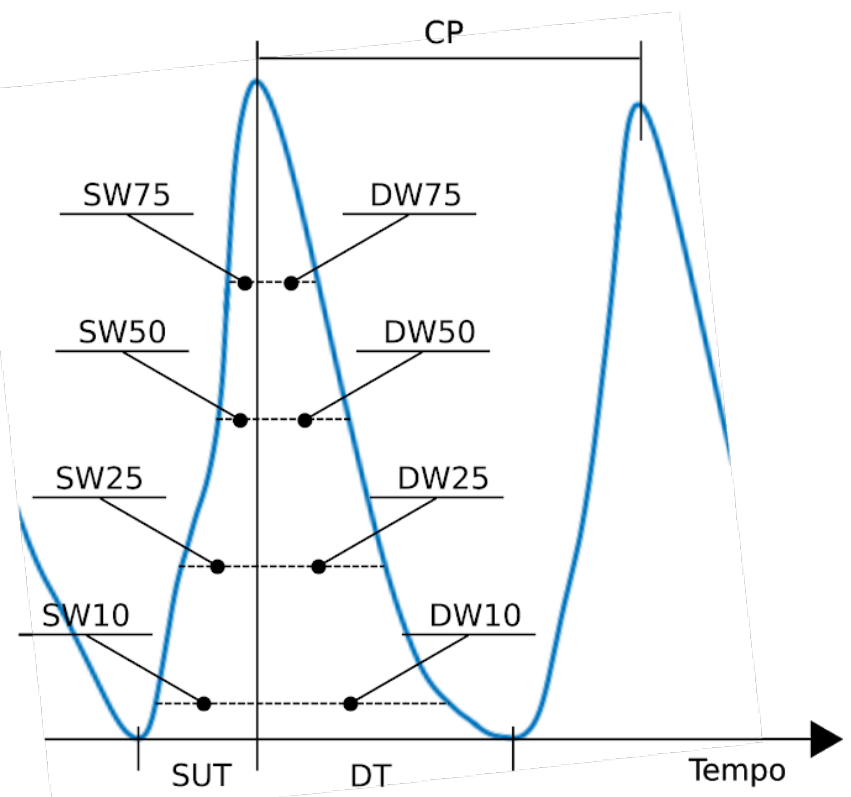

Fig. 2. Esquema de Características Extraídas da Onda PPG

imos estão abaixo do terceiro quartil. Entre dois pontos de máximo deve existir apenas um ponto de mínimo. Segundo Zhang and Feng (2017), o período cardíaco de um paciente corresponde ao intervalo de tempo entre dois pontos de máximo consecutivos $(\mathrm{CP})$. O intervalo entre um ponto de máximo e o ponto de mínimo seguinte corresponde ao tempo diastólico (DT), enquanto o intervalo entre o ponto de mínimo e o ponto de máximo seguinte corresponde ao tempo de subida sistólico (SUT). A largura do pulso da onda, tomando como referência o seu pico, são chamados pelo autor de largura sistólica (SW) e largura diastólica (DW). DW50 e SW50, por exemplo, representam o equivalente ao intervalo entre o ponto correspondente a $50 \%$ da amplitude e o instante de tempo correspondente ao pico. O esquema apresentado na Figura 3.1.2 apresenta graficamente como os valores são extraídos.

Os valores extraídos das ondas, e utilizados como valores de entrada na RNA, foram CP, SUT, DT, CP*SUT, CP*DT, DW10, SW10, DW25, SW25, DW33, SW33, DW50, SW50, DW66, SW66, DW75 e SW75. Adicional- 


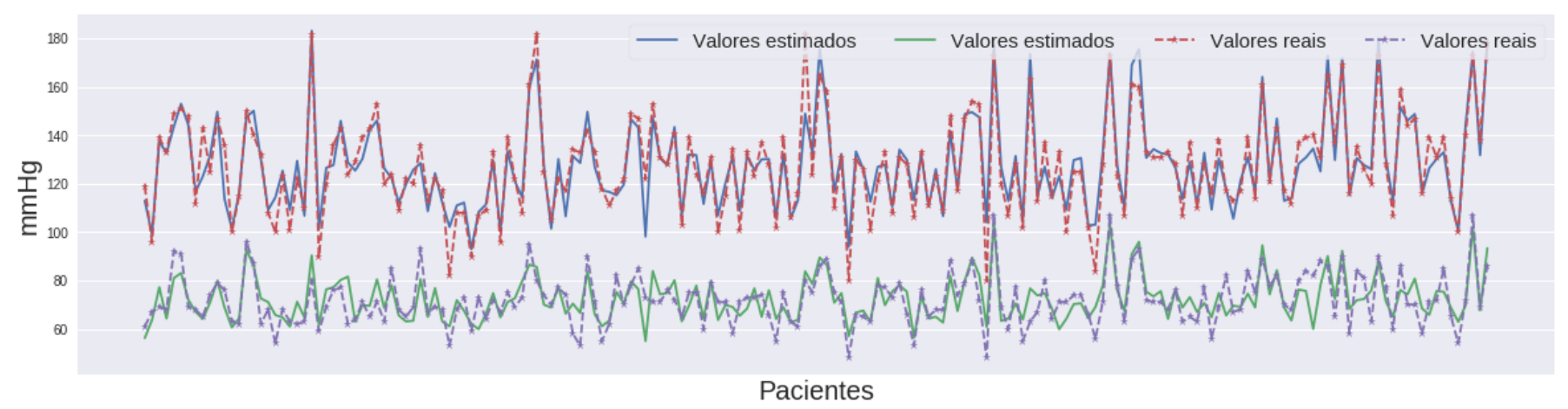

Fig. 3. Comparação dos resultados dos dados de teste ( Índice da amostra vs. BP normalizado )

mente aos dados acima, foram fornecidas a idade, altura, peso, sexo e informação de tipo de enfermidade que, por ventura, o paciente possa sofrer. As citadas patologias são: hipertensão, diabetes, infarto e acidente vascular cerebral (AVC). No total, são 25 parâmetros de entrada na rede. Com estes valores, deseja-se obter, a partir de um modelo, os valores de pressão sistólica e diastólica de um paciente. Estes valores são medidos pela quantidade milímetros de Mercúrio $(\mathrm{mmHg})$.

\subsection{Método de Regressão}

Uma rede neural do tipo feed-forward é constituída por camadas, normalmente, uma camada de entrada, uma ou mais camadas escondidas e uma camada de saída. Essas redes neurais (HAYKIN, 2001) são chamadas de perceptron de múltiplas camadas (do inglês, Multi Layer Perceptron - MLP), as quais representam uma generalização do perceptron de camada única.

O funcionamento de um a rede MLP é síncrono, ou seja, dada uma entrada (um vetor) esta é propagada para a saída multiplicando-se pelos pesos de cada camada, aplicando-se uma função de ativação para cada camada e propagando esse valor para a camada seguinte até que a camada de saída seja alcançada.

Tais redes têm sido aplicadas com sucesso para resolver diversos problemas complexos, através do seu treinamento de forma supervisionada com um algoritmo popular conhecido como algoritmo de retropropagação, também referenciado na literatura como back-propagation (HAYKIN, 2001).

O método empregado para o aprendizado e predição da variáveis de interesse foi a rede Peceptron de Múltiplas Camadas utilizando backward propagation para atualização do pesos.

A arquitetura da MLP aplicada neste problema possui 3 camadas, sendo: camada de entrada com 15 neurônios e relu como função de ativação; camada intermediária com 10 neurônios e função tanh e camada de saída com 2 neurônios e ativação linear. Os valores iniciais para os pesos são gerados aleatoriamente, a partir de uma distribuição uniforme no intervalo $[-1,1]$.

\section{RESULTADOS}

O modelo definido na seção anterior foi implementado em Python utilizando a biblioteca Keras. A Figura 3 mostra os dados de teste. Nela, as informações estão representadas como:

- linha tracejada vermelha - medição da pressão sanguínea sistólica (SBP);

- linha azul - estimação da pressão sanguínea sistólica;

- linha tracejada roxa - medição da pressão sanguínea diastólica (DBP);

- linha verde - estimação da pressão sanguínea diastólica.

Enquanto a Figura 4 mostra os histogramas do erro entre a medição e o valor estimado pelo preditor $\left(\right.$ erro $\left.=y_{\text {test }}-\hat{y}\right)$.

A Figura 4 apresenta: Histograma dos dados de medição (SBP em azul e DBP em verde); Histograma dos dados da predição (SBP em azul e DBP em verde).

Foram realizadas 1000 execuções da RNA na configuração apresentada na Seção 3 para estimar a variância e o erro médio, frente àqueles conjuntos de dados. Estes são escolhidos aleatoriamente a cada execução numa proporção 70/30: $70 \%$ para o conjunto de treinamento e o restante, 30 $\%$, para o conjunto de testes. O número de execuções foi definido empiricametne. Nessas condições, forma obtidos os resultados a seguir:

- Média do valor de erro médio: $6.57 \mathrm{mmHg}$;

- Média sistólica: $5.63 \mathrm{mmHg}$;

- Média diastólica: $5.37 \mathrm{mmHg}$;

- Variância da média: $0.11 \mathrm{mmHg}$;

- Variância sistólica: $11.90 \mathrm{mmHg}$

- Variância diastólica: $10.88 \mathrm{mmHg}$.

Outras arquiteturas com diferentes números de camadas, número de neurônios e diferentes funções de ativação foram testadas, antes de se alcançar estes resultados.

\section{CONCLUSÕES}

Os resultados obtidos mostram que a metodologia de préprocessamento e extração de características do sinal PPG é adequada e promissora. Ao final, a diferença entre os valores previstos pelo modelo e a pressão real disponível na base de dados encontram-se dentro dos limites estabelecidos por normas internacionais (Ogedegbe and Pickering, 

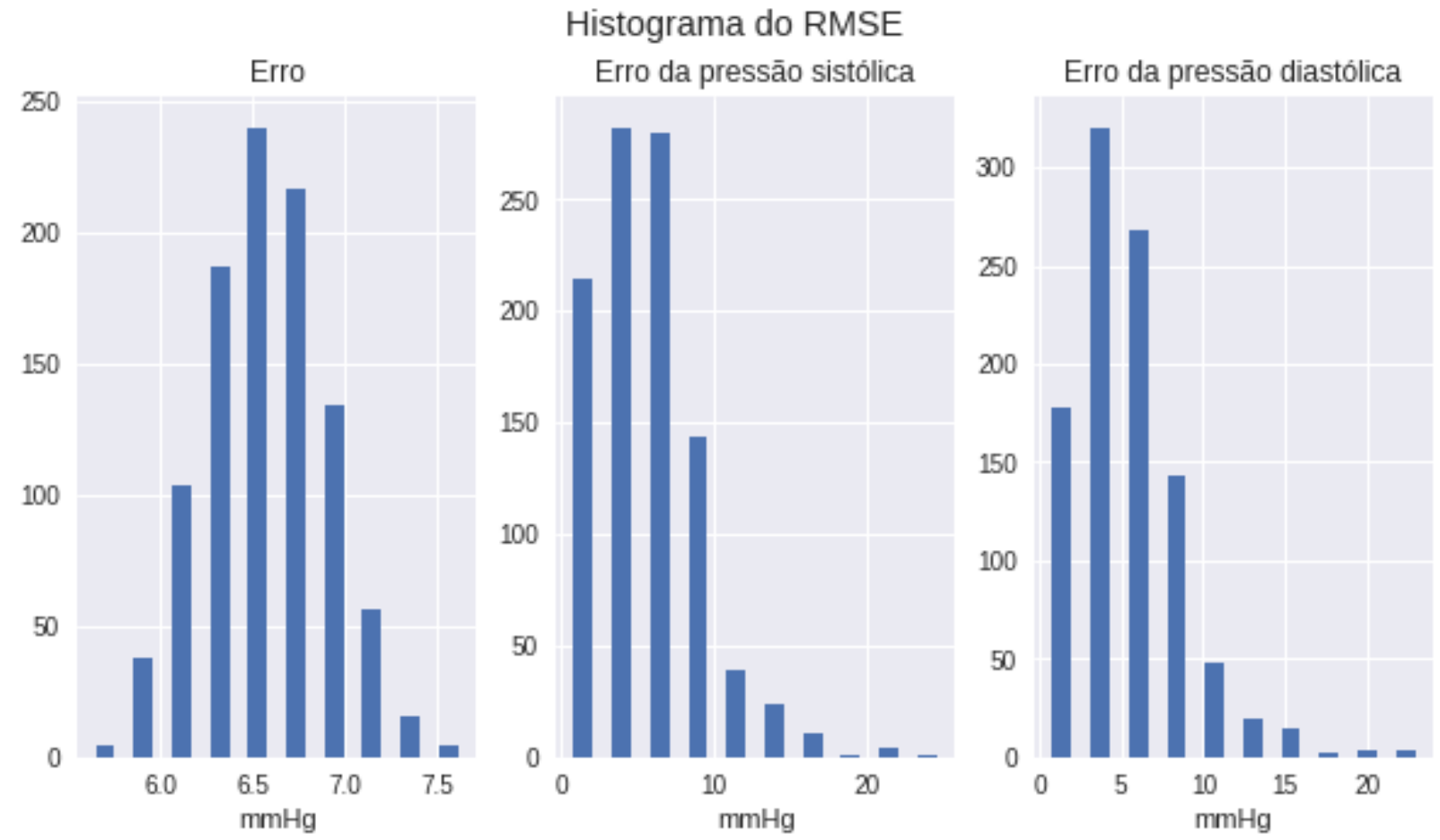

Fig. 4. Histograma do erro entre o valor estimado e o valor medido para: (1) SBP e DBP; (2) SBP; (3) DBP
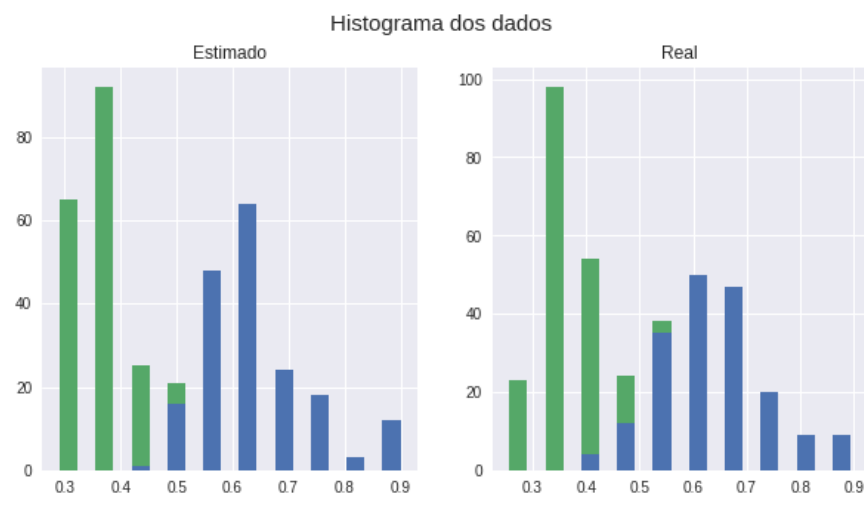

Fig. 5. Comparação dos dados de medição e predição

2010). Contudo, existe a possibilidade de melhoria nos resultados, sobretudo na variância do erro. Um diferencial deste trabalho é que foi utilizado no emprego da RNA uma base de dados detalhada e que difere de outras disponíveis publicamente. Nesta, a pressão sistólica e diastólica contém um valor único, enquanto noutras, investigadas até aqui, trata-se de uma informação intrísica em formato de onda. O treinamento da rede neural foi satisfatório e a predição no conjunto de testes mostrou-se com boa precisão.

Como desdobramento deste trabalho, pode-se pensar em análises estatísticas para determinação do fator de contribuição de cada característica na acurácia do modelo. Outra intenção de pesquisas futuras seria investigar meios para se obter simplificação na extração destes dados e possibilitar treinar o modelo com um número menor de iterações e de parâmetros, reduzindo o custo computacional.

\section{REFERENCES}

Allen, J. (2007). Photoplethysmography and its application in clinical physiological measurement. Physiological Measurement, 28(3), R1-R39. doi:10.1088/0967-3334/ $28 / 3 / \mathrm{r} 01$.

Banegas, J.R., Ruilope, L.M., de la Sierra, A., Vinyoles, E., Gorostidi, M., de la Cruz, J.J., Ruiz-Hurtado, G., Segura, J., Rodríguez-Artalejo, F., and Williams, B. (2018). Relationship between clinic and ambulatory blood-pressure measurements and mortality. New England Journal of Medicine, 378(16), 1509-1520.

Bronzino, J. and Peterson, D. (2014). Biomedical Engineering Fundamentals. The Biomedical Engineering Handbook, Fourth Edition. CRC Press.

Corrêa, L.A.F. (2006). Sistema Não Invasivo de Monitorização da Pressão Arterial e da Onda de Pulso Utilizando a Fotoplestismografia. mathesis, Universidade Federal do Rio de Janeiro, Rio de Janeiro.

da Cunha, S.B. and Carvajal, S. (2009). Estatistica Básica - A Arte de Trabalhar com Dados. Elsevier Brasil.

de Andrade, P.B., Rinaldi, F.S., de Castro Bienert, I.R., Barbosa, R.A., Bergonso, M.H., de Matos, M.P.B., de Matos Ferreira, C.F.B., Nogueira, E.F., Labrunie, A., Kreimer, S., et al. (2015). Fatores de risco para complicações relacionadas ao acesso vascular em pacientes submetidos à estratégia invasiva precoce. Revista Brasileira de Cardiologia Invasiva, 23(4), 242-246.

Gunasekaran, V. (2013). Continuous Non-Invasive Arterial Blood Pressure Measurement Using Photoplethysmography. mathesis, UC San Diego, San Diego.

HAYKIN, S. (2001). Redes Neurais. BOOKMAN COMPANHIA ED, 2nd edition.

Kamshilin, A.A. and Margaryants, N.B. (2017). Origin of photoplethysmographic waveform at green light. 
Physics Procedia, 86, 72-80. doi:10.1016/j.phpro.2017. 01.024.

Kurylyak, Y., Lamonaca, F., and Grimaldi, D. (2013). A neural network-based method for continuous blood pressure estimation from a PPG signal. In 2013 IEEE International Instrumentation and Measurement Technology Conference (I2MTC). IEEE. doi:10.1109/i2mtc. 2013.6555424.

Lamelas, P. and Jolly, S. (2015). Identificação de pacientes com risco de complicações do acesso vascular. Rev. bras. cardiol. invasiva, 23(4), f-236.

Liang, Y., Chen, Z., Liu, G., and Elgendi, M. (2018). A new, short-recorded photoplethysmogram dataset for blood pressure monitoring in china. Scientific Data, 5, 180020. doi:10.1038/sdata.2018.20.

Ogedegbe, G. and Pickering, T. (2010). Principles and techniques of blood pressure measurement. Cardiology Clinics, 28(4), 571-586. doi:10.1016/j.ccl.2010.07.006.

Song, S., Cho, J., Oh, H., Lee, J., and Kim, I. (2009). Estimation of blood pressure using photoplethysmography on the wrist. In 2009 36th Annual Computers in Cardiology Conference (CinC), 741-744. Park City, UT.

Wang, W., den Brinker, A.C., Stuijk, S., and de Haan, G. (2017). Algorithmic principles of remote PPG. IEEE Transactions on Biomedical Engineering, 64(7), 14791491. doi:10.1109/tbme.2016.2609282.

WHO (2018). Cardiovascular diseases (cvds). http://www. who.int/en/news-room/fact-sheets/ detail/cardiovascular-diseases-(cvds).

Xing, X. and Sun, M. (2016). Optical blood pressure estimation with photoplethysmography and FFT-based neural networks. Biomedical Optics Express, 7(8), 3007. doi:10.1364/boe.7.003007.

Yongbo Liang, Guiyong Liu, Zhencheng Chen, and Elgendi, M. (2017). Ppg-bp database. https:// figshare.com/articles/PPG-BP_Database_zip/ 5459299. doi:10.6084/M9.FIGSHARE.5459299.

Zhang, Y. and Feng, Z. (2017). A SVM method for continuous blood pressure estimation from a PPG signal. In Proceedings of the 9th International Conference on Machine Learning and Computing - ICMLC 2017. ACM Press. doi:10.1145/3055635.3056634. 